\title{
Disentangling Standard Model EFT operators with future low-energy parity-violating electron scattering experiments
}

\author{
Radja Boughezal, ${ }^{1}$ Frank Petriello, ${ }^{1,2}$ and Daniel Wiegand $\oplus^{1,2}$ \\ ${ }^{1}$ HEP Division, Argonne National Laboratory, Argonne, Illinois 60439, USA \\ ${ }^{2}$ Department of Physics \& Astronomy, Northwestern University, Evanston, Illinois 60208, USA
}

(Received 19 April 2021; accepted 14 June 2021; published 7 July 2021)

\begin{abstract}
We study the potential of future parity-violating electron scattering (PVES) data to probe the parameter space of the Standard Model effective field theory. We contrast the constraints derived from Drell-Yan data taken at the Large Hadron Collider (LHC) with projections of the planned PVES experiments SoLID and P2. We show that the PVES data can complement the bounds set by the LHC data in the dimension-six operator space since it probes different combinations of operators than Drell-Yan. The lower characteristic energy of P2 and SoLID also helps disentangle effects of dimension-six and dimension-eight operators that are difficult to resolve with LHC Drell-Yan data alone.
\end{abstract}

DOI: $10.1103 /$ PhysRevD.104.016005

\section{INTRODUCTION}

No conclusive sign of physics beyond the Standard Model (SM) has been observed at either the Large Hadron Collider (LHC) or in other experiments. This has motivated significant attention toward studying how potential extensions of the SM are constrained by the current data. Understanding the implications of current measurements will provide insight on both the energy scale at which new physics can appear and to what sectors of the SM new heavy particles are allowed to couple given the existing constraints.

A consistent framework to perform such an analysis, with the assumption that any new physics is significantly heavier than the electroweak scale, is the SM effective field theory (SMEFT). The SMEFT is constructed by augmenting the SM Lagrangian with higher-dimensional operators consistent with the SM gauge symmetries and formed only from SM fields. The higher-dimensional operators in the SMEFT are suppressed by appropriate powers of a characteristic energy scale $\Lambda$ below which heavy new fields are integrated out. Complete, nonredundant bases for the dimension-six [1-3] and dimension-eight operators $[4,5]$ have been identified. Odd-dimensional operators violate the lepton number and will not be considered in this work.

It is an ongoing effort to analyze the numerous available data within the SMEFT framework, primarily in partial

Published by the American Physical Society under the terms of the Creative Commons Attribution 4.0 International license. Further distribution of this work must maintain attribution to the author(s) and the published article's title, journal citation, and DOI. Funded by SCOAP . analysis of individual SMEFT sectors [6-16]. Recent work has been devoted to performing a global, simultaneous fit of all data available [17-25]. Most of these global fits have focused on the truncation of the SMEFT expansion to dimension-six operators. Several open issues must be confronted when performing such analyses. When performing a global fit with a greater numbers of parameters than observables, only certain linear combinations of the fit parameters can be probed. These flat directions in parameter space can be either exact or approximate in the sense that they hold only in certain kinematic limits. Large deviations from the SM, as measured by turning on SMEFT operators with sizeable Wilson coefficients, can lead to no observable consequences in the presence of such degeneracies. Finding observables that remove these flat directions is an important component of improving the efficacy of global fits to the SMEFT. Another issue to address is the sensitivity of fits to dimension-eight and higher operators. Intuitively their effects should be suppressed, but since many measurements at the LHC probe high energies this assumption must be tested. Furthermore, dimension-eight effects sometimes represent the leading SMEFT contributions in models with certain approximate symmetries [26,27]. Previous analyses of the impact of dimension-eight operators can be found in the literature [28-35].

The goal of this paper is to investigate the potential impact of high-luminosity, low-energy parity violating electron scattering (PVES) experiments in resolving both issues discussed above. Our work is motivated by the planned SoLID experiment at Jefferson Laboratory [36,37] as well as the planned P2 experiment at MESA [38]. These two low energy experiments are complementary to each other since, due to the nature of the targets used, they probe 
different combinations of Wilson coefficients. Our analysis focuses on semileptonic four-fermion operators at both dimension-six and dimension-eight. Previous analyses [14] of PVES data in terms of the asymmetry parameter $A_{\mathrm{PV}}$ focused on constraining dimension-six modifications of the electroweak couplings. Certain combinations of dimension-six semileptonic four-fermion operators are already well probed by high invariant mass Drell-Yan distributions at the LHC $[14,39,40]$. In principle the available LHC data should also be able to constrain the corresponding dimension-eight operators due to the large integrated luminosity that has been collected as well as the sufficiently large center of mass energy. In practice the Drell-Yan process exhibits numerous flat directions that complicates the separation of different dimension-six effects [40,41], as well the disentanglement of dimension-six from dimension-eight operators as we show later in this paper. The considerably lower energy of the PVES experiments leads to a suppression of dimension-eight effects, and therefore sensitivity to dimension-six operators only. Combining LHC with SoLID and P2 respectively allows these different order operators to be disentangled. We furthermore show that PVES experiments can be used to lift flat directions in the space of dimension-six operators when combined with Drell-Yan data. Our work follows in the spirit of previous analyses that showed how future data from an electron-ion collider (EIC) could help resolve degeneracies present in SMEFT fits using Drell-Yan data only [41]. One advantage of the SoLID and P2 experiments is that they are anticipated to start data taking within the next few years, as opposed to the longer time frame of the EIC.

Our paper is organized as follows: In Sec. II we review the aspects of the SMEFT framework relevant for our analysis. We present and discuss the formulas describing the Drell-Yan process at the LHC and parity-violating scattering at SoLID and P2 in Sec. III. In Sec. IV we present the main results of our paper, combined fits of the DrellYan data with SoLID and P2 projections, and illustrate their potential to differentiate between both dimension- 6 and dimension-8 operators. Finally, in Sec. V we put our findings in perspective and conclude.

\section{NOTATION AND SMEFT FORMALISM}

We review in this section aspects of the SMEFT relevant for our analysis of LHC and projected PVES data. The SMEFT is an extension of the SM Lagrangian including terms suppressed by an energy scale $\Lambda$ at which the ultraviolet completion is assumed to become important and new particles beyond the SM appear. Truncating the expansion in $1 / \Lambda$ at dimension eight, and ignoring operators of odd dimension which violate the lepton number, we have

$$
\mathcal{L}=\mathcal{L}_{\mathrm{SM}}+\frac{1}{\Lambda^{2}} \sum_{i} C_{i}^{6} \mathcal{O}_{6, i}+\frac{1}{\Lambda^{4}} \sum_{i} C_{i}^{8} \mathcal{O}_{8, i}+\ldots
$$

TABLE I. Dimension-six operators that potentially contribute to the Drell-Yan process and their relevant dimension-eight extensions. We refer to the dimension-eight operators in this table as "type-1". The corresponding "type-2" operators are not shown.

\begin{tabular}{lccc}
\hline \hline \multicolumn{2}{c}{ Dimension Six } & \multicolumn{2}{c}{ Dimension Eight } \\
\hline $\mathcal{O}_{l q}^{(1)}$ & $\left(\bar{l} \gamma^{\mu} l\right)\left(\bar{q} \gamma_{\mu} q\right)$ & $\mathcal{O}_{l^{2} q^{2} D^{2}}^{(1)}$ & $D^{\nu}\left(\bar{l} \gamma^{\mu} l\right) D_{\nu}\left(\bar{q} \gamma_{\mu} q\right)$ \\
$\mathcal{O}_{l q}^{(3)}$ & $\left(\bar{l} \gamma^{\mu} \tau^{i} l\right)\left(\bar{q} \gamma_{\mu} \tau^{i} q\right)$ & $\mathcal{O}_{l^{2} q^{2} D^{2}}$ & $D^{\nu}\left(\bar{l} \gamma^{\mu} \tau^{i} l\right) D_{\nu}\left(\bar{q} \gamma_{\mu} \tau^{i} q\right)$ \\
$\mathcal{O}_{e u}$ & $\left(\bar{e} \gamma^{\mu} e\right)\left(\bar{u} \gamma_{\mu} u\right)$ & $\mathcal{O}_{e^{2} \nu^{2} D^{2}}$ & $D^{\nu}\left(\bar{e} \gamma^{\mu} e\right) D_{\nu}\left(\bar{u} \gamma_{\mu} u\right)$ \\
$\mathcal{O}_{e d}$ & $\left(\bar{e} \gamma^{\mu} e\right)\left(\bar{d} \gamma_{\mu} d\right)$ & $\mathcal{O}_{e^{2} d^{2} D^{2}}$ & $D^{\nu}\left(\bar{e} \gamma^{\mu} e\right) D_{\nu}\left(\bar{d} \gamma_{\mu} d\right)$ \\
$\mathcal{O}_{l u}$ & $\left(\bar{l} \gamma^{\mu} l\right)\left(\bar{u} \gamma_{\mu} u\right)$ & $\mathcal{O}_{l^{2} u^{2} D^{2}}^{(1)}$ & $D^{\nu}\left(\bar{l} \gamma^{\mu} l\right) D_{\nu}\left(\bar{u} \gamma_{\mu} u\right)$ \\
$\mathcal{O}_{l d}$ & $\left(\bar{l} \gamma^{\mu} l\right)\left(\bar{d} \gamma_{\mu} d\right)$ & $\mathcal{O}_{l^{2} d^{2} D^{2}}^{(1)}$ & $D^{\nu}\left(\bar{l} \gamma^{\mu} l\right) D_{\nu}\left(\bar{d} \gamma_{\mu} d\right)$ \\
$\mathcal{O}_{q e}$ & $\left(\bar{q} \gamma^{\mu} q\right)\left(\bar{e} \gamma_{\mu} e\right)$ & $\mathcal{O}_{q^{2} e^{2} D^{2}}^{(1)}$ & $D^{\nu}\left(\bar{q} \gamma^{\mu} q\right) D_{\nu}\left(\bar{e} \gamma_{\mu} e\right)$ \\
\hline \hline
\end{tabular}

where the ellipsis denotes operators of higher dimensions. The Wilson coefficients $C_{i}^{d}$ defined above are dimensionless. We calculate cross sections to leading order in the coupling constants as well as to dimension eight in the SMEFT expansion. This includes contributions from both true dimension-eight operators as well as contributions of dimension-six operators squared. For both SoLID and P2 observables we have explicitly checked that dimension-eight contributions are suppressed like $Q^{2} / \Lambda^{4}$, where $Q^{2}<$ $6 \mathrm{GeV}^{2}$ is the energy transfer relevant for the SoLID and P2 experiments. Since the SMEFT requires $\Lambda$ to be much greater than the electroweak scale all dimension-eight effects are completely negligible for PVES kinematics. For notational simplicity we will drop the explicit dimension labels on the operators and Wilson coefficients when no confusion between them can occur.

In Table I we compile the operators that affect the DrellYan and PVES processes at leading order in the coupling constants. We have used the notation of Ref. [4] for the dimension-eight operators. We assume massless fermions as well as minimal flavor violation (MFV) for the SMEFT Wilson coefficients. With this assumption the interference between SM and SMEFT amplitudes scales as the corresponding fermion mass divided by the new physics scale, which we assume to be a negligible effect. Though the dimension-six squared effect is not a priori mass suppressed, it scales again with the SM Yukawa couplings when assuming MFV since the Wilson coefficients become proportional to the Yukawa couplings in this case. It is therefore also negligible. We note at this point that for each dimension-six operator there are two dimension-eight extensions that differ in the placement of the covariant derivatives. We will refer to those compiled in Table I as "type-1" operators. The other possibility, which we will call "type-2" can schematically be written as $\left(\bar{\psi} \gamma^{\mu} \stackrel{\leftrightarrow}{D^{\nu}} \psi\right)\left(\bar{\psi} \gamma_{\mu} \stackrel{\leftrightarrow}{D_{\nu}} \psi\right)$ in terms of the left-right derivative 
$\stackrel{\leftrightarrow}{D^{\mu}}=\overrightarrow{D^{\mu}}-\overleftarrow{D^{\mu}}$. As we will explain in greater detail later the type-1 operators lead to only an energy-dependent shift of the corresponding dimension-six effects, while the type-2 operators lead to a different angular dependence. The effect of the type- 2 dimension-eight extensions can therefore in principle be disentangled through angular variables [32] and we omit them from our analyses. In Table I $l$ and $q$ denote $S U(2)$ lepton and quark doublets respectively, while $e, u$ and $d$ are the right-handed singlets. $\tau^{i}$ are the $S U(2)$ Pauli matrices and $D_{\mu}$ is the covariant derivative. We suppress flavor indices for notational clarity. Though our analysis of PVES data is only sensitive to first-generation operators it is necessary to disentangle contributions from different generations when preparing a global fit of all operators. A possible strategy in the leptonic sectors was presented in Ref. [42]. The Wilson coefficients are in principle dependent on the renormalization scheme chosen. In an $\overline{\mathrm{MS}}$ scheme they become scale-dependent and run with energy. As we perform only a leading-order analysis in this work we neglect this running.

Historically it has been customary to parametrize the parity-violating, dimension-six interactions in terms of the following phenomenological four-fermion Lagrangian [43]:

$$
\begin{aligned}
\mathcal{L}_{\mathrm{PV}}= & \frac{G_{F}}{\sqrt{2}}\left[\left(\bar{e} \gamma^{\mu} \gamma_{5} e\right)\left(C_{1 u}^{6} \bar{u} \gamma_{\mu} u+C_{1 d}^{6} \bar{d} \gamma_{\mu} d\right)+\left(\bar{e} \gamma^{\mu} e\right)\left(C_{2 u}^{6} \bar{u} \gamma_{\mu} \gamma_{5} u+C_{2 d}^{6} \bar{d} \gamma_{\mu} \gamma_{5} d\right)\right. \\
& +\left(\bar{e} \gamma^{\mu} e\right)\left(C_{V u}^{6} \bar{u} \gamma_{\mu} u+C_{V d}^{6} \bar{d} \gamma_{\mu} d\right)+\left(\bar{e} \gamma^{\mu} \gamma_{5} e\right)\left(C_{A u}^{6} \bar{u} \gamma_{\mu} \gamma_{5} u\right) \\
& +D^{\nu}\left(\bar{e} \gamma^{\mu} \gamma_{5} e\right) D_{\nu}\left(\frac{C_{1 u}^{8}}{v^{2}} \bar{u} \gamma_{\mu} u+\frac{C_{1 d}^{8}}{v^{2}} \bar{d} \gamma_{\mu} d\right)+D^{\nu}\left(\bar{e} \gamma^{\mu} e\right) D_{\nu}\left(\frac{C_{2 u}^{8}}{v^{2}} \bar{u} \gamma_{\mu} \gamma_{5} u+\frac{C_{2 d}^{8}}{v^{2}} \bar{d} \gamma_{\mu} \gamma_{5} d\right) \\
& \left.+D^{\nu}\left(\bar{e} \gamma^{\mu} e\right) D_{\nu}\left(\frac{C_{V u}^{8}}{v^{2}} \bar{u} \gamma_{\mu} u+\frac{C_{V d}^{8}}{v^{2}} \bar{d} \gamma_{\mu} d\right)+D^{\nu}\left(\bar{e} \gamma^{\mu} \gamma_{5} e\right) D_{\nu}\left(\frac{C_{A u}^{8}}{v^{2}} \bar{u} \gamma_{\mu} \gamma_{5} u\right)\right] .
\end{aligned}
$$

We have extended this parametrization to include the type-1 dimension-eight extensions of the usual operators. We will refer to this as the PVES basis in this paper. The dimension-six portion of this phenomenological operator basis can be mapped onto the usual dimension-six SMEFT basis via the transformation

$$
\begin{aligned}
& C_{1 u}^{6}=2\left(g_{R}^{e}-g_{L}^{e}\right)\left(g_{R}^{u}+g_{L}^{u}\right)+\frac{v^{2}}{2 \Lambda^{2}}\left\{-\left(C_{l q}^{(1)}-C_{l q}^{(3)}\right)+C_{e u}+C_{q e}-C_{l u}\right\} \\
& C_{2 u}^{6}=2\left(g_{R}^{e}+g_{L}^{e}\right)\left(g_{R}^{u}-g_{L}^{u}\right)+\frac{v^{2}}{2 \Lambda^{2}}\left\{-\left(C_{l q}^{(1)}-C_{l q}^{(3)}\right)+C_{e u}-C_{q e}+C_{l u}\right\} \\
& C_{1 d}^{6}=2\left(g_{R}^{e}-g_{L}^{e}\right)\left(g_{R}^{d}+g_{L}^{d}\right)+\frac{v^{2}}{2 \Lambda^{2}}\left\{-\left(C_{l q}^{(1)}+C_{l q}^{(3)}\right)+C_{e d}+C_{q e}-C_{l d}\right\} \\
& C_{2 d}^{6}=2\left(g_{R}^{e}+g_{L}^{e}\right)\left(g_{R}^{d}-g_{L}^{d}\right)+\frac{v^{2}}{2 \Lambda^{2}}\left\{-\left(C_{l q}^{(1)}+C_{l q}^{(3)}\right)+C_{e d}-C_{q e}+C_{l d}\right\} \\
& C_{V u}^{6}=2\left(g_{R}^{e}+g_{L}^{e}\right)\left(g_{R}^{u}+g_{L}^{u}\right)+\frac{v^{2}}{2 \Lambda^{2}}\left\{\left(C_{l q}^{(1)}-C_{l q}^{(3)}\right)+C_{e u}+C_{q e}+C_{l u}\right\} \\
& C_{A u}^{6}=2\left(g_{R}^{e}-g_{L}^{e}\right)\left(g_{R}^{u}-g_{L}^{u}\right)+\frac{v^{2}}{2 \Lambda^{2}}\left\{\left(C_{l q}^{(1)}-C_{l q}^{(3)}\right)+C_{e u}-C_{q e}-C_{l u}\right\} \\
& C_{V d}^{6}=2\left(g_{R}^{e}+g_{L}^{e}\right)\left(g_{R}^{d}+g_{L}^{d}\right)+\frac{v^{2}}{2 \Lambda^{2}}\left\{\left(C_{l q}^{(1)}+C_{l q}^{(3)}\right)+C_{e d}+C_{q e}+C_{l d}\right\} .
\end{aligned}
$$

The dimension-eight part of the phenomenological operator basis can be mapped through the same transformation with the dimension-six SMEFT coefficients replaced by their dimension-eight counterparts in Table I and an additional factor of $\frac{v^{2}}{\Lambda^{2}}$. We additionally note that the coefficients of $\mathcal{L}_{\mathrm{PV}}$ have nonzero SM values unlike the SMEFT coefficients, due to the inclusion of SM gauge-boson exchanges in their definitions. For the dimension-eight transformation the SM offset is scaled by $\frac{v^{2}}{M_{Z}^{2}}$. These shifts can be expressed in terms of the left- and right-handed fermion couplings to the $Z$ boson. The leading-order values for these form factors follow the conventions in [44] and amount to

$$
g_{L}^{f}=I_{3}^{f}-Q_{f} s_{W}^{2}, \quad g_{R}^{f}=-Q_{f} s_{W}^{2}, \quad g_{Z}=\frac{e}{s_{W} c_{W}} .
$$


Finally, we note that the axial-axial down-type operators with coefficients $C_{A d}^{6}$ and $C_{A d}^{8}$ are omitted from the Lagrangian in Eq. (2). We will see later that the use of this basis helps reveal experimental sensitivity to specific ultraviolet completions of the SMEFT that are obscure in the SMEFT basis for four-fermion Wilson coefficients.

The basis of dimension-six semileptonic four-fermion SMEFT operators is built from $S U(2)$ doublets and singlets and consists of seven independent operators after electroweak symmetry breaking. Naïvely one would expect eight operators making up $\mathcal{L}_{\mathrm{PV}}$. This basis is over complete since it is formed from fields after electroweak symmetry breaking, and we can eliminate one operator by making use of the underlying $S U(2)$ symmetry.

\section{REVIEW OF DRELL-YAN AND PVES FORMULAS}

In this section we review the formulas describing the Drell-Yan process and the parity-violating asymmetry parameter $A_{\mathrm{PV}}$ in PVES. The review of the Drell-Yan cross sections closely follows Ref. [41].

\section{A. Review of Drell-Yan}

We first present the cross section for the partonic Drell-Yan process $q+\bar{q} \rightarrow e^{+}+e^{-}$. We decompose the differential cross section into three SM pieces stemming from photon and $Z$-boson exchange and their interference, two terms for interference between SM and SMEFT for each of the dimension-six and dimension-eight operators respectively and one piece for the SMEFT dimension-six squared term,

$$
\begin{aligned}
\frac{d \sigma_{q \bar{q}}}{d m_{l l}^{2} d Y d c_{\theta}}= & \frac{1}{32 \pi m_{l l}^{2} \hat{s}} f_{q}\left(x_{1}\right) f_{\bar{q}}\left(x_{2}\right)\left\{\frac{d \hat{\sigma}_{q \bar{q}}^{\gamma \gamma}}{d m_{l l}^{2} d Y d c_{\theta}}+\frac{d \hat{\sigma}_{q \bar{q}}^{\gamma Z}}{d m_{l l}^{2} d Y d c_{\theta}}+\frac{d \hat{\sigma}_{q \bar{q}}^{Z Z}}{d m_{l l}^{2} d Y d c_{\theta}}\right. \\
& \left.+\frac{d \hat{\sigma}_{q \bar{q}}^{\gamma \mathrm{SMEFT} 6}}{d m_{l l}^{2} d Y d c_{\theta}}+\frac{d \hat{\sigma}_{q \bar{q}}^{Z \mathrm{SMEFT} 6}}{d m_{l l}^{2} d Y d c_{\theta}}+\frac{d \hat{\sigma}_{q \bar{q}}^{\gamma \mathrm{SMEFT}}}{d m_{l l}^{2} d Y d c_{\theta}}+\frac{d \hat{\sigma}_{q \bar{q}}^{Z \mathrm{SMEFT} 8}}{d m_{l l}^{2} d Y d c_{\theta}}+\frac{d \hat{\sigma}_{q \bar{q}}^{\mathrm{SMEFT} 6^{2}}}{d m_{l l}^{2} d Y d c_{\theta}}\right\}
\end{aligned}
$$

Here the $x_{i}$ are the partonic momentum fractions and $f_{q}(x)$ is the parton distribution function describing the probability of finding a parton $q$ of momentum fraction $x$ inside the proton. $m_{l l}$ is the invariant mass of the two final state leptons and $Y$ is its rapidity. Finally, $c_{\theta}$ is the cosine of the center of mass scattering angle of the negatively charged lepton. The hadronic cross section for the Drell-Yan process is derived by summing over all possible initial state quarks found inside the proton and integrating over their momentum fractions $x_{1}$ and $x_{2}$. The explicit expressions for the three terms making up the SM contribution to the differential cross section for initial state up-type quarks are given by

$$
\begin{aligned}
\frac{d \hat{\sigma}_{u \bar{u}}^{\gamma \gamma}}{d m_{l l}^{2} d Y d c_{\theta}} & =\frac{32 \pi^{2} \alpha^{2} Q_{u}^{2}}{3} \frac{\hat{t}^{2}+\hat{u}^{2}}{\hat{s}^{2}}, \\
\frac{d \hat{\sigma}_{u \bar{u}}^{\gamma Z}}{d m_{l l}^{2} d Y d c_{\theta}} & =-\frac{8 \pi \alpha Q_{u} g_{Z}^{2}}{3} \frac{\left(g_{R}^{u} g_{L}^{e}+g_{R}^{e} g_{L}^{u}\right) \hat{t}^{2}+\left(g_{R}^{u} g_{R}^{e}+g_{L}^{e} g_{L}^{u}\right) \hat{u}^{2}}{\hat{s}\left(\hat{s}-M_{Z}^{2}\right)} \\
\frac{d \hat{\sigma}_{u \bar{u}}^{Z Z}}{d m_{l l}^{2} d Y d c_{\theta}} & =\frac{g_{Z}^{4}}{3} \frac{\left(\left(g_{R}^{u} g_{L}^{e}\right)^{2}+\left(g_{R}^{e} g_{L}^{u}\right)^{2}\right) \hat{t}^{2}+\left(\left(g_{R}^{u} g_{R}^{e}\right)^{2}+\left(g_{L}^{e} g_{L}^{u}\right)^{2}\right) \hat{u}^{2}}{\left(\hat{s}-M_{Z}^{2}\right)^{2}} .
\end{aligned}
$$

The interference terms between the SM and the leading dimension-six contributions is

$$
\begin{aligned}
\frac{d \hat{\sigma}_{u \bar{u}}^{\gamma \mathrm{SMEFT} 6}}{d m_{l l}^{2} d Y d c_{\theta}} & =-\frac{8 \pi \alpha Q_{u}}{3 \Lambda^{2}} \frac{\left(C_{l u}+C_{q e}\right) \hat{t}^{2}+\left(C_{e u}+C_{l q}^{(1)}-C_{l q}^{(3)}\right) \hat{u}^{2}}{\hat{s}} \\
\frac{d \hat{\sigma}_{u \bar{u}}^{Z \mathrm{SMEFT} 6}}{d m_{l l}^{2} d Y d c_{\theta}} & =\frac{2 g_{Z}^{2}}{3 \Lambda^{2}} \frac{\left(g_{R}^{u} g_{L}^{e} C_{l u}+g_{R}^{e} g_{L}^{u} C_{q e}\right) \hat{t}^{2}+\left(g_{R}^{u} g_{R}^{e} C_{e u}+g_{L}^{u} g_{L}^{e} C_{l q}^{(1)}-g_{L}^{u} g_{L}^{e} C_{l q}^{(3)}\right) \hat{u}^{2}}{\hat{s}-M_{Z}^{2}}
\end{aligned}
$$

The contributions stemming from interference of the SM with the corresponding dimension-eight terms, as well as the dimension-six squared pieces, are 


$$
\begin{aligned}
\frac{d \hat{\sigma}_{u \bar{u}}^{\gamma \mathrm{SMEFT}}}{d m_{l l}^{2} d Y d c_{\theta}}= & -\frac{8 \pi \alpha Q_{u}}{3 \Lambda^{4}}\left[\left(C_{l^{2} u^{2} D^{2}}^{(1)}+C_{q^{2} e^{2} D^{2}}^{(1)}\right) \hat{t}^{2}+\left(C_{e^{2} u^{2} D^{2}}^{(1)}+C_{l^{2} q^{2} D^{2}}^{(1)}-C_{l^{2} q^{2} D^{2}}^{(3)}\right) \hat{u}^{2}\right] \\
\frac{d \hat{\sigma}_{u \bar{u}}^{Z \text { SMEFT } 8}}{d m_{l l}^{2} d Y d c_{\theta}}= & \frac{2 g_{Z}^{2}}{3 \Lambda^{4}} \frac{\hat{s}}{\hat{s}-M_{Z}^{2}}\left[\left(g_{R}^{u} g_{L}^{e} C_{l^{2} u^{2} D^{2}}^{(1)}+g_{R}^{e} g_{L}^{u} C_{q^{2} e^{2} D^{2}}^{(1)}\right) \hat{t}^{2}\right. \\
& \left.+\left(g_{R}^{u} g_{R}^{e} C_{e^{2} u^{2} D^{2}}^{(1)}+g_{L}^{u} g_{L}^{e} C_{l^{2} q^{2} D^{2}}^{(1)}-g_{L}^{u} g_{L}^{e} C_{l^{2} q^{2} D^{2}}^{(3)}\right) \hat{u}^{2}\right] \\
\frac{d \hat{\sigma}_{u \bar{u}}^{\mathrm{SMEFT} 6^{2}}}{d m_{l l}^{2} d Y d c_{\theta}}= & \frac{1}{3 \Lambda^{4}}\left[\left(C_{l u}^{2}+C_{q e}^{2}\right) \hat{t}^{2}+\left(C_{e u}^{2}+\left(C_{l q}^{(1)}-C_{l q}^{(3)}\right)^{2}\right) \hat{u}^{2}\right] .
\end{aligned}
$$

These depend on the standard Mandelstam variables $\hat{s}, \hat{t}$ and $\hat{u}$. The down-type contributions to the cross section can be found by replacing the indices and Wilson coefficients as follows:

$$
\begin{aligned}
Q_{u} & \rightarrow Q_{d} \quad g_{L / R}^{u} \rightarrow g_{L / R}^{d} \quad C_{l q}^{(3)} \rightarrow-C_{l q}^{(3)} \quad C_{l u} \rightarrow C_{l d} \quad C_{e u} \rightarrow C_{e d} \\
C_{l^{2} u^{2} D^{2}}^{(1)} & \rightarrow C_{l^{2} d^{2} D^{2}}^{(1)} \quad C_{e^{2} u^{2} D^{2}}^{(1)} \rightarrow C_{e^{2} d^{2} D^{2}}^{(1)} \quad C_{l^{2} q^{2} D^{2}}^{(3)} \rightarrow-C_{l^{2} q^{2} D^{2}}^{(3)}
\end{aligned}
$$

\section{B. Review of PVES}

The SoLID parity-violating deep-inelastic scattering (PVDIS) experiment will scatter a $12 \mathrm{GeV}$ polarized electron beam from polarized proton or deuteron targets $[36,45,46]$. Similarly P2 will scatter a $155 \mathrm{MeV}$ electron beam elastically from hydrogen and carbon targets [38]. A goal of both experiments is to measure the parity-violating asymmetry

$$
A_{\mathrm{PV}}=\frac{\sigma_{R}-\sigma_{L}}{\sigma_{R}+\sigma_{L}}
$$

where the $R$ and $L$ subscripts refer to incoming left- and right-handed electrons respectively. SoLID measurements will be taken in the kinematic range of $2 \mathrm{GeV}^{2}<Q^{2}<$ $10 \mathrm{GeV}^{2}$ and $x>0.2$. The data taken using the proton target will be used to further constrain the $d / u$ ratio in the proton while the data taken with the deuteron target will be used to constrain new physics. $\mathrm{P} 2$ is dedicated to extracting a precise low-energy measurement of the electroweak mixing angle from $A_{\mathrm{PV}}$. Since the electroweak mixing angle is predicted by the Standard Model, its precise measurement can be used to constrain new physics that shifts its value from the SM prediction.

In the limit $Q^{2} \ll M_{Z}^{2}$ the asymmetry parameter can be written as $[47,48]$

$$
A_{\mathrm{PV}}=-\left(\frac{G_{F} Q^{2}}{4 \sqrt{2} \pi \alpha}\right)^{2}\left[Y_{1}\left(x, y, Q^{2}\right) a_{1}+Y_{3}\left(x, y, Q^{2}\right) a_{3}\right]
$$

with

This is under the assumption that the parity-violating interactions are parametrized by the phenomenological Lagrangian in Eq. (2). The kinematic dependencies are encoded in the $Y_{i}$ functions:

$$
\begin{aligned}
& Y_{1}\left(x, y, Q^{2}\right)=\frac{1+(1-y)^{2}-y^{2}\left(1-r^{2} /\left(1+R^{\gamma Z}\right)\right)-2 x y M / E}{1+(1-y)^{2}-y^{2}\left(1-r^{2} /\left(1+R^{\gamma}\right)\right)-2 x y M / E}\left(\frac{1+R^{\gamma Z}}{1+R^{\gamma}}\right), \\
& Y_{3}\left(x, y, Q^{2}\right)=\frac{1-(1-y)^{2}}{1+(1-y)^{2}-y^{2}\left(1-r^{2} /\left(1+R^{\gamma}\right)\right)-2 x y M / E}\left(\frac{r^{2}}{1+R^{\gamma}}\right),
\end{aligned}
$$


which in turn depend on $r=1+\frac{4 M^{2} x^{2}}{Q^{2}}$, the electroweak structure functions $R^{i}=R^{i}\left(x, Q^{2}\right)$ as well as the target mass $M$ and beam energy $E$. These formulas are the basis for the projections of how well SoLID [45] and P2 [38] will be able to constrain the parity-violating coefficients $C_{i q}$. Due to the scalar nature of the carbon nucleus used in the P2 experiment, the measurement will be insensitive to the $C_{2 q}$ coefficients and will put bounds on $C_{1 q}$. SoLID is expected to be more sensitive to $C_{2 q}$.

We wish to emphasize at this point that it is important to analyze data from the LHC and PVES experiments in both the traditional SMEFT basis and also using the $C_{1 q}$ and $C_{2 q}$ coefficients instead. Since these coefficients map to a mix of SMEFT Wilson coefficients it is tempting to simply consider the resulting bounds in the SMEFT basis only. However, some ultraviolet completions, for example those with a leptophobic $Z^{\prime}[49,50]$ only lead to a modification of the $C_{2 q}$ while the $C_{1 q}$ retain their SM values. The search for $Z^{\prime}$ bosons is an ongoing effort at the LHC [51] and it is important to be able to clearly interpret which regions of parameter space have been excluded by other experiments. While these results can be recovered in the SMEFT basis by choosing the appropriate combinations of Wilson coefficients, they are more easily seen in the basis consisting of the $C_{1 q}$ and $C_{2 q}$.

\section{COMPLEMENTARITY BETWEEN LHC DRELL_YAN DATA AND PVES}

In order to illustrate the impact that both SoLID and P2 have on breaking the degeneracies present in the Drell-Yan data we consider four example scenarios.

(a) We first reproduce the SoLID projection of Ref. [45] as well as the P2 projection of Ref. [38] in the PVES basis of Eq. (2). We study the constraints imposed by LHC Drell-Yan data in this parameter space and analyze the impact of combining the results of these experiments.

(b) We next consider the SMEFT basis and turn on only dimension-six operators. We consider the combined fits of Wilson-coefficient combinations for which the Drell-Yan data has been previously shown to exhibit flat directions [41], and study how SoLID and P2 can lift these degeneracies.

(c) We turn on both dimension-six and dimension-eight operators in the basis of Eq. (2). In this basis it is easy to see that SoLID is uniquely capable of disentangling coefficients of type $C_{2 q}$ while $\mathrm{P} 2$ probes coefficients of type $C_{1 q}$.

(d) Finally we turn on both dimension-six and dimensioneight operators in the SMEFT basis. We show that LHC Drell-Yan measurements can only poorly differentiate certain dimension-six operators from dimension-eight effects, and then show that these degeneracies are lifted by PVES $A_{\mathrm{PV}}$ measurements.
For the analysis of the Drell-Yan process we follow Ref. [41] and use the ATLAS data set presented in [52] (since it both goes to high invariant mass and is measured to high precision). We use the fully-correlated experimental errors. The SoLID collaboration anticipates using the data obtained from a hydrogen target to better determine the $d / u$ ratio inside the proton. Measurements taken with deuteron targets are dedicated to BSM searches. The SoLID projection [45] is based on the assumption that the data available will stem from a single bin $0.4<x<0.5$ taken at $Q^{2}=6 \mathrm{GeV}^{2}$ with a $12 \mathrm{GeV}$ electron beam. ${ }^{1}$ Measurements from the other available bins will serve to keep the systematic error under control. The total error for the measurement, including statistical uncertainties as well as both experimental and theoretical systematic errors, is predicted to be $0.6 \%$. The $\mathrm{P} 2$ projection [38] assumes that the data stems from the scattering of a $150 \mathrm{MeV}$ polarized electron beam off hydrogen and carbon targets. It is assumed that the electroweak mixing angle extracted from these measurements will have a $0.3 \%$ relative error, stemming mostly from the uncertainty of the beam polarization. This projection furthermore includes already determined constraints from SLAC [53], the atomic parity violation in Cesium [54], as well as the anticipated bounds from the QWeak experiment [55].

To quantify the deviation from SM predictions we define a $\chi^{2}$ test statistic according to

$$
\chi^{2}=\sum_{i, j}^{\# \text { of observables }} \frac{\left(\sigma_{i}^{\mathrm{SM}}-\sigma_{i}^{\mathrm{SMEFT}}\right)\left(\sigma_{j}^{\mathrm{SM}}-\sigma_{j}^{\mathrm{SMEFT}}\right)}{\Delta \sigma_{i j}^{2}} .
$$

For both the SoLID and P2 experiments there is no data available yet, so we project the SM to be correct and the SMEFT to measure simply the deviation from the SM allowed by the data. The sum over observables included all Drell-Yan invariant mass bins from Ref. [52] as well as the SoLID measurements and all observables making up the P2 projection.

We note that the Wilson coefficients are in principle dependent on the renormalization scheme chosen and are therefore subject to renormalization group running [5658]. The measurements at LHC are taken at higher scales than the low-energy experiments. However, since we are performing an analysis to demonstrate the power of the PVES experiments before the availability of the actual data we neglect these effects here. They can be straightforwardly included once the data is available.

\section{A. Dimension-six analysis in the $\mathcal{L}_{\mathrm{PV}}$ basis}

We begin by considering the constraints in the traditional basis used for PVES in Eq. (2). Both P2 and SoLID are

\footnotetext{
${ }^{1}$ We thank K. Kumar and P. Souder for discussions on how to reproduce the SoLID projections.
} 


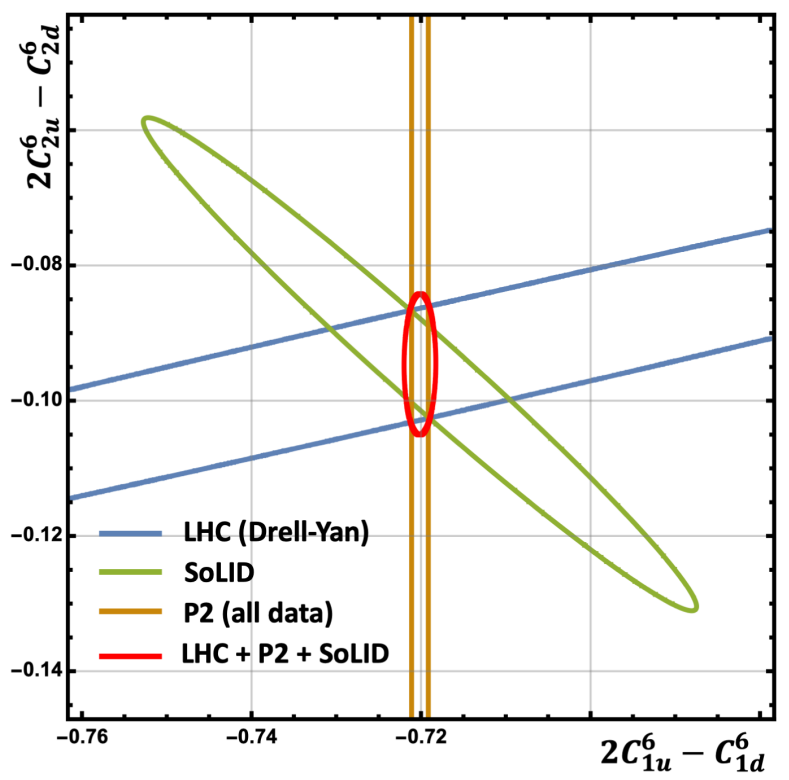

FIG. 1. Combination of the $68 \%$ C.L. bounds derived from Drell-Yan data, P2 and SoLID in the dimension-six $C_{i u} / C_{i d}$ basis. Note the nonzero SM at the center of the ellipses. It corresponds to the loop-corrected first terms of Eq. (3) $[59,60]$. In the case of P2 we include projections for data taken with both hydrogen and carbon targets and a projection for the QWeak experiment as well as available data from atomic-parity violation.

designed to access different combinations of PVES coefficients. P2 will constrain $2 C_{1 u}^{6}+C_{1 d}^{6}$ through the hydrogen measurements as well as the combination $C_{1 u}^{6}+C_{1 d}^{6}$ with the carbon target data. The anticipated bounds on the individual coefficients $C_{1 i}^{6}$ are therefore expected to be tight enough to be neglected in the SoLID analysis. SoLID is thus expected to be able to focus on setting bounds on the combination $2 C_{2 u}^{6}-C_{2 d}^{6}$. To illustrate this point in our analysis, and compare with the available Drell-Yan data we set only the combinations of parameters $2 C_{1 u}-C_{1 d}$ and $2 C_{2 u}-C_{2 d}$ to be nonzero. The orthogonal combinations of parameters are assumed to vanish. The same combinations of parameters can be arranged for the Drell-Yan cross section using the conversion of Eq. (3). We note that all dimension-eight operators are turned off for this case. Similarly we neglect contributions from squared dimension-six operators which are formally of the same order. Using the $\chi^{2}$ function defined above we study the constraints from SoLID, from P2, from LHC Drell-Yan data, and from the combination of all three experiments. As a check of our analysis procedure we note that our SoLID constraint ellipse matches that of Ref. [45]. Our results are shown in Fig. 1. The constraint ellipses associated with the SoLID and LHC measurements are nearly orthogonal, indicating that the directions poorly probed by Drell-Yan will be strongly constrained by SoLID. This is shown explicitly by the combined ellipse in Fig. 1, which features a significantly smaller allowed parameter space. The P2 constraints are strong but can only probe the $C_{1 q}$ direction. We note that only the SoLID experiment can simultaneously probe both directions in the $2 C_{1 u}^{6}-C_{1 d}^{6}$ versus $2 C_{2 u}^{6}-C_{2 d}^{6}$ space, and will consequently provide an important check of any combined LHC and P2 constraints.

\section{B. Dimension-six analysis in the SMEFT basis}

We now switch to the SMEFT basis. To illustrate the constraining power these low-energy experiments have in the space of SMEFT coefficients, we investigate example Wilson coefficient choices that lead to flat directions in the parameter space. The choice of these examples is adapted from Ref. [41].

\section{Dimension-six: Case 1}

In a first scenario we assume $C_{e u}, C_{q e}$ and $C_{e d}$ to be nonzero and truncate all matrix elements at order $\frac{1}{\Lambda^{2}}$. We see from Eq. (7) that these coefficients appear in both terms containing $\hat{t}$ as well as terms proportional to $\hat{u}$. In principle there should not be a flat direction. This is however not the case when analyzing the currently available high-invariant mass LHC data [41]; after performing the angular integrations relevant for the $m_{l l}$ distributions the discriminatory power in the angular distributions vanishes. After performing the angular integration we find that the SMEFT contributions vanishes for

$C_{e d}=\frac{Q_{u} e^{2}-g_{Z}^{2} g_{L}^{u} g_{R}^{e}}{Q_{u} e^{2}-g_{Z}^{2} g_{R}^{e} g_{R}^{u}} \frac{Q_{d} e^{2}-g_{Z}^{2} g_{R}^{e} g_{R}^{d}}{Q_{d} e^{2}-g_{Z}^{2} g_{L}^{d} g_{R}^{e}} C_{e u} \equiv C_{e d}^{(2)}$.

We perform a two-dimensional $\chi^{2}$ fit after projecting $C_{e d}$ down to $C_{e d}^{(2)}$. The constraints are shown in Fig. 2. Due to the flat direction the constraints derived from Drell-Yan data are fairly loose and only constrain the absolute values of $C_{e u}$ and $C_{q e}$ to be smaller than about 15 and 40 respectively (we normalize the operators to $\Lambda=3 \mathrm{TeV}$ and limit the plot to the relevant region in which the contours intersect). Including projected SoLID data removes this flat direction since PVES is proportional to different combinations of SMEFT parameters. With both LHC and SoLID the same coefficients are constrained to about 1 and 4.

The P2 experiment will be able to constrain this combination of parameters even further. $\mathrm{P} 2$, shown by the brown ellipse in Fig. 2, constrains $\left|C_{q e}\right|$ to smaller than about 0.2 and $\left|C_{e u}\right|$ to smaller than 0.4. This serves to illustrate an important point that generically appears when analyzing PVES constraints in the SMEFT basis. The $C_{1 q}$ coefficients are linear combinations of all relevant SMEFT coefficients, as evident from Eq. (3). At the same time the anticipated constraints from the $\mathrm{P} 2$ experiments on $C_{1 q}$ are extremely strong, with an expected precision on certain linear combinations of the $C_{1 q}$ approaching a few times 


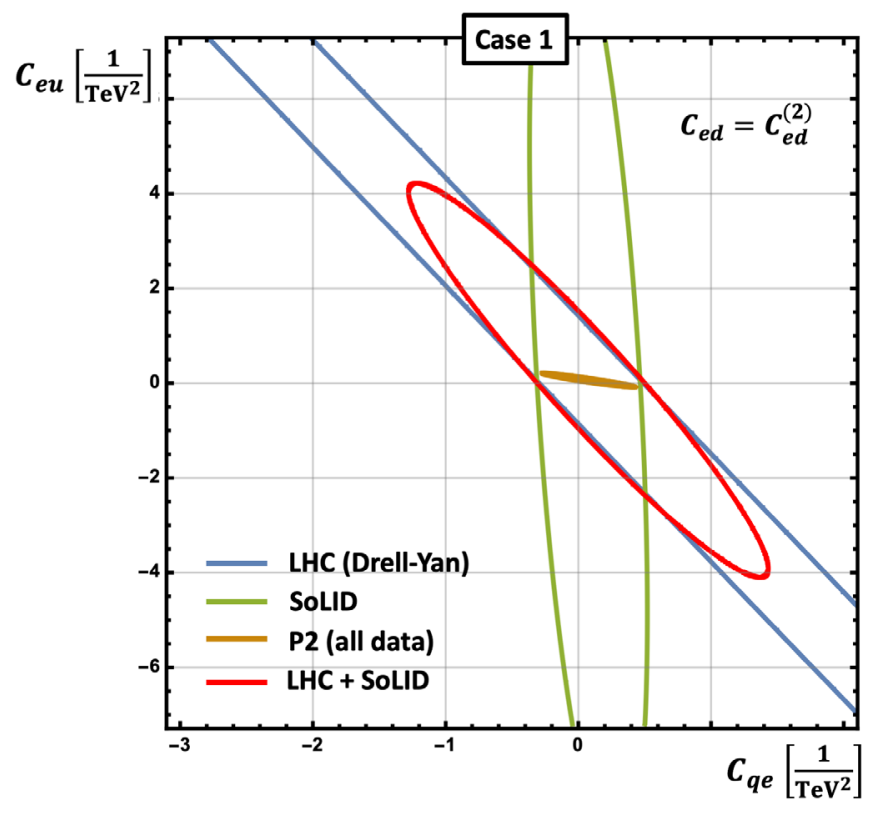

FIG. 2. Combining the $68 \%$ C.L. bounds derived from DrellYan data for the Case 1 scenario combined with the projections for P2 and SoLID in the SMEFT basis. The operators are normalized to $\Lambda=3 \mathrm{TeV}$.

0.001 [38]. In contrast the expected SoLID constraints on the $C_{2 q}$ are on the order of a few times 0.01 . Since a generic SMEFT Wilson coefficient projects onto both $C_{1 q}$ and $C_{2 q}$ the constraints from $\mathrm{P} 2$ will be far stronger than those from SoLID on those coefficients. The power of the SoLID experiment is only revealed when certain linear combinations of SMEFT coefficients are considered, such as those motivated by leptophobic $Z^{\prime}$ ultraviolet completions $[49,50]$. Although the physics is basis independent, the results are sometimes easier to see in a certain basis, and this is a strong motivation to consider constraints in both the SMEFT and PVES bases when analyzing these experiments.

\section{Dimension-6: Case 2}

We next assume that $C_{l q}^{(1)}, C_{e u}$ and $C_{e d}$ are nonzero. As discussed in detail in Ref. [41] these coefficients only contribute to the $\hat{u}^{2}$ term in Eq. (7). In the high-invariant mass limit we can arrange these coefficients so that the correction to the Drell-Yan cross section vanishes. The particular combination of dimension-six SMEFT contributions that leads to this is [41]

$$
C_{e d}=\frac{Q_{u} e^{2}-g_{Z}^{2} g_{R}^{u} g_{R}^{e}}{Q_{u} e^{2}-g_{Z}^{2} g_{L}^{e} g_{L}^{u}} \frac{Q_{d} e^{2}-g_{Z}^{2} g_{L}^{e} g_{L}^{d}}{Q_{d} e^{2}-g_{Z}^{2} g_{R}^{d} g_{R}^{e}} C_{e u} \equiv C_{e d}^{(1)},
$$

indicating a flat direction in the dimension-six parameter space. We again illustrate the bounds the Drell-Yan data can set with a two-dimensional fit in Fig. 3 where we project down onto the flat direction $C_{e d}=C_{e d}^{(1)}$. We contrast the

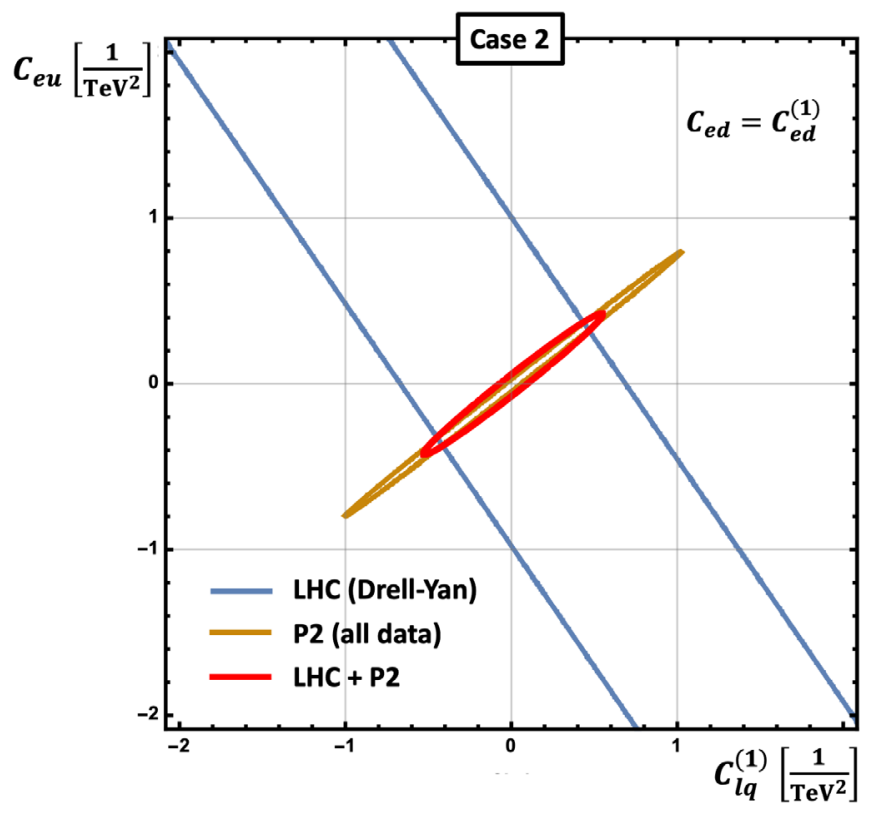

FIG. 3. Combining the $68 \%$ C.L. bounds derived from DrellYan data for the Case 2 scenario combined with the projections for SoLID and P2 in the SMEFT basis. The operators are normalized to $\Lambda=3 \mathrm{TeV}$.

LHC bounds with the projected P2 bounds. As discussed in the previous section the expected constraints from SoLID are weaker, and we do not show them explicitly here. The Drell-Yan analysis only manages to constrain the absolute values of $C_{l q}^{(1)}$ and $C_{e u}$ to be smaller than 30 and 40 respectively. Combining the two measurements reduces the allowed dimension-six parameter space considerably. At $68 \%$ confidence we can constrain both Wilson coefficients well below unity.

\section{Dimension-6: Case 3}

Finally we consider one more combination of dimension-six SMEFT coefficients. In order to explore a larger swath of the dimension-six parameter space we turn on the up-type operators $C_{l u}$ and $C_{e u}$. This serves to illustrate a scenario in which probes from all three experiments become comparable. In this example we can also show the potential of combining the experiments even if the observables do not suffer from flat directions, which is the case for this choice of Wilson coefficients.

After performing the two-dimensional $\chi^{2}$ fit we arrive at the bounds shown in Fig. 4. A fit to the Drell-Yan data is able to constrain $\left|C_{l u}\right|$ and $\left|C_{e u}\right|$ to be smaller than about 15 and 10 respectively. SoLID constrains the same coefficients to $\left|C_{l u}\right|<5$ and $\left|C_{e u}\right|<0.5$. P2 exhibits a flat direction and can only constrain the combination $\left|C_{l u}-C_{e u}\right|$ to be smaller than 0.08. Combining all three measurements enables us to dramatically cut down on the available parameter space and allows us to constrain the absolute value of each individual coefficient to be smaller than 0.5 . 


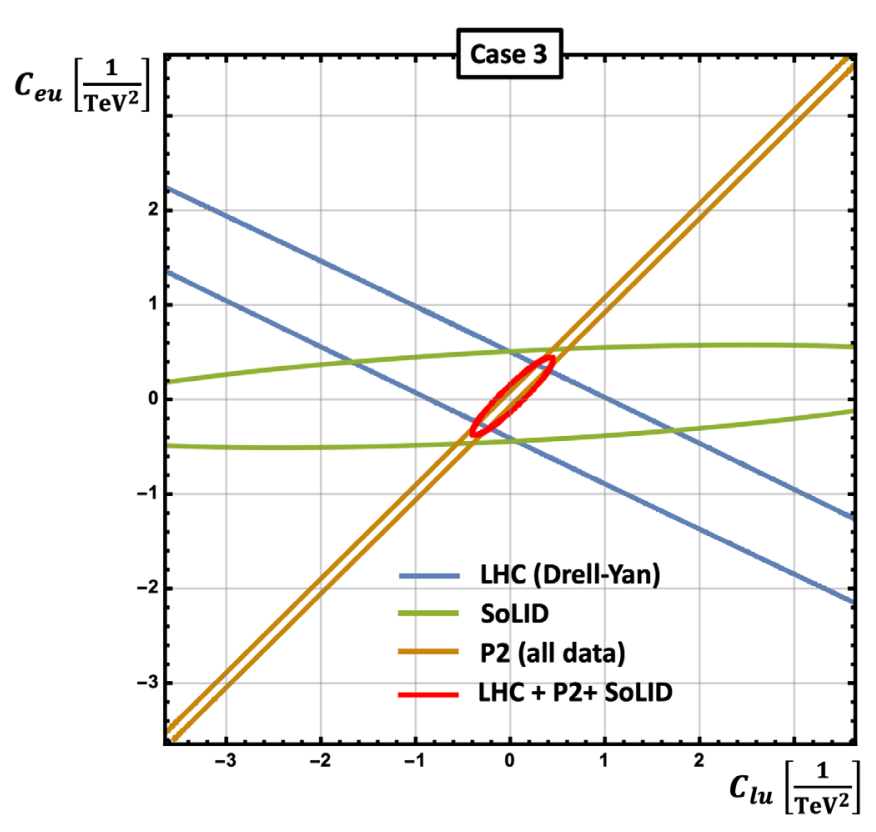

FIG. 4. Combining the $68 \%$ C.L. bounds derived from DrellYan data for the Case 3 scenario combined with the projections for P2 and SoLID in the SMEFT basis. The operators are normalized to $\Lambda=3 \mathrm{TeV}$.

\section{Joint dimension-six and dimension-eight fits in the $\mathcal{L}_{\mathrm{PV}}$ basis}

We now study the effect of simultaneously turning on both dimension-six and dimension-eight operators. For consistency of the EFT expansion we also include the square of dimension-six coefficients in our calculations. Naively we would expect that measurements of the invariant mass distribution at the LHC would distinguish between dimension-six and dimension- 8 effects since they scale differently with energy. However, we find significant interplay between the dimension-six squared effects and the dimension-eight operators, leading to degeneracies in the parameter space. Both SoLID and P2 help to resolve these degeneracies. As low-energy experiments, they have completely negligible dependence on $\mathcal{O}\left(1 / \Lambda^{4}\right)$ effects, and are therefore sensitive to only $\mathcal{O}\left(1 / \Lambda^{2}\right)$ dimension-six effects. To illustrate how SoLID and P2 complement each other in constraining the parameter space we first perform an analysis in the PVES basis defined by Eq. (2). We begin by turning on the combination $2 C_{1 u}-C_{1 d}$ and its dimension-eight extension $2 C_{1 u}^{8}-C_{1 d}^{8}$ for both $\mathrm{P} 2$ and Drell-Yan at LHC. As mentioned previously the $\mathrm{P} 2$ probes of the $C_{1 q}$ coefficients are stronger than the SoLID probes of these terms. We limit ourselves to the two-derivative extension discussed in Sec. II. The placement of the derivatives leads to an overall energy-dependent scaling of the dimension-six term. Performing a fit to the LHC data leads to the blue contour in Fig. 5. The contour is no longer a simple ellipse due to the presence of dimension-six squared terms. The $1 \sigma$ contour for P2 is shown in brown and does not exhibit a

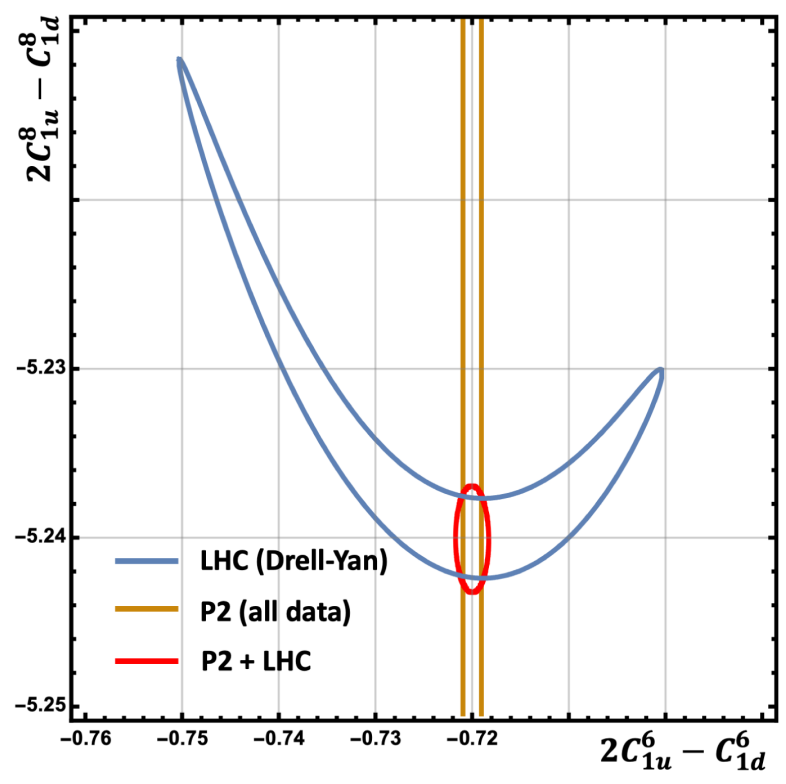

FIG. 5. Combining the $68 \%$ C.L. bounds derived from DrellYan data and the $\mathrm{P} 2$ projection in the $C_{i u} / C_{i d}$ basis contrasting dimension-six and dimension-eight contributions.

dependence on the dimension-eight operators, as expected. P2 significantly reduces the parameter space allowed by the LHC Drell-Yan data. The elongation of the LHC bounds occurs due to cancellations between dimension-eight effects and dimension-six squared terms. These can be removed by $\mathrm{P} 2$ measurements.

We can probe the other scenario where instead the $C_{2 q}$ coefficients are turned on, and the SoLID probes are more important than $\mathrm{P} 2$ ones. We consider the scenario where $2 C_{2 u}^{6}-C_{2 d}^{6}$ and its dimension-eight extension $2 C_{2 u}^{8}-C_{2 d}^{8}$ are turned on. The resulting bounds for Drell-Yan at LHC and SoLID in terms of these operators are shown in Fig. 6. Again, the low-energy PVDIS experiment SoLID can rule out parameter space allowed by the LHC, although the effects are not as significant as in the previous P2 analysis.

\section{Joint dimension-six and dimension-eight fits in the SMEFT basis}

To illustrate how the inclusion of low-energy PVES experiments impacts the bounds on SMEFT coefficients we now translate the fits of the previous section into the SMEFT basis. As an example we consider the type- 1 dimension-eight operator $C_{l^{2} q^{2} D^{2}}^{(1)}$, which we see from Table I is a two-derivative extension of the dimensionsix term $C_{l q}^{(1)}$. The resulting bounds in terms of the SMEFT operators are shown in Fig. 7. The stretching of the constraint contour occurs because the SMEFT modification of the Drell-Yan cross section vanishes for the combination

$$
C_{l^{2} q^{2} D^{2}}^{(1)}=C_{l q}^{(1)}\left[\frac{1}{2} \frac{C_{l q}^{(1)}}{e^{2} Q_{u}-g_{z}^{2} g_{L}^{e} g_{L}^{u}}-\frac{\Lambda^{2}}{\hat{s}}\right]
$$




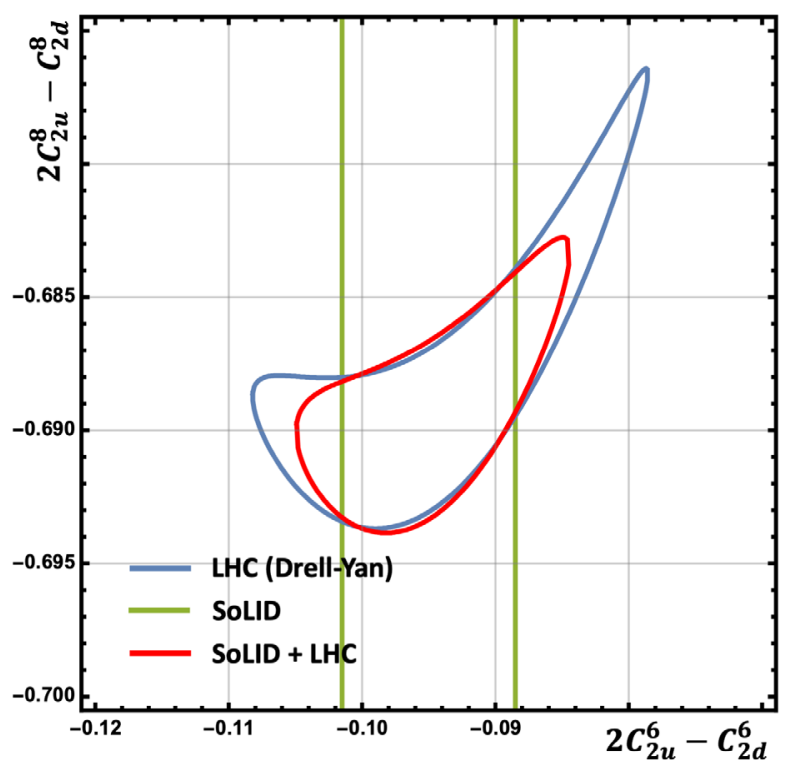

FIG. 6. Combining the $68 \%$ C.L. bounds derived from DrellYan data and the SoLID projection in the $C_{i u} / C_{i d}$ basis contrasting dimension-six and dimension-eight contributions.

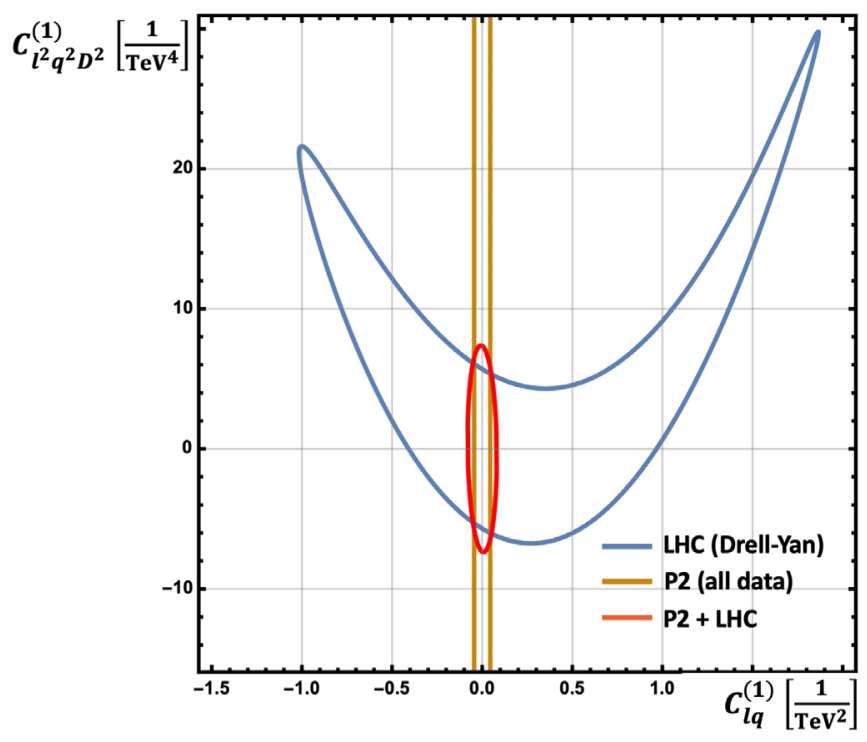

FIG. 7. Example SMEFT plot combining the $68 \%$ C.L. bounds derived from Drell-Yan data and the $\mathrm{P} 2$ projection for $C_{l q}^{(1)}$ and its type-1 dimension-eight extension $C_{l^{2} q^{2} D^{2}}^{(1)}$. The operators are normalized to $\Lambda=3 \mathrm{TeV}$.

in the high-energy limit $s \gg M_{Z}^{2}$. Although this condition changes as the invariant mass bin changes, most sensitivity comes from the higher invariant mass bins, leading to the long tails seen in the plot. The asymmetry parameter $A_{\mathrm{PV}}$ is, in principle, dependent on the same linear combination of coefficients. However, the dimension-eight piece is suppressed by $Q^{2} / \Lambda^{2}$, and the $\mathrm{P} 2$ projection is therefore largely independent of $C_{l^{2} q^{2} D^{2}}^{(1)}$. Combining the Drell-Yan bounds with the projected $\mathrm{P} 2$ results constrains $\left|C_{l q}^{(1)}\right|$ to be less than 0.1 , while $\left|C_{l^{2} q^{2} D^{2}}^{(1)}\right|$ is bound to be smaller than 8 .

\section{CONCLUSIONS}

In this work we have studied the potential impact that future PVES experiments SoLID and P2 will have on disentangling degeneracies in SMEFT fits, and in separating dimension-six from dimension-eight effects. Both experiments can discriminate between combinations of dimension-six operators that cannot be resolved by existing Drell-Yan data at the LHC. We have studied several such examples motivated by previous work [41] to demonstrate this point. A generic issue that we have discussed extensively in this work is the importance of studying Wilsoncoefficient bases motivated by specific ultraviolet examples in order to properly evaluate the impact of different experiments. In the situation here the use of the traditional PVES basis in terms of $C_{1 q}$ and $C_{2 q}$ illustrates complementarity between the SoLID and P2 experiments difficult to see in the SMEFT basis. We have illustrated through the use of the PVES basis that the bounds on parameter space set by SoLID and P2 are complementary and how these bounds translate into the standard SMEFT basis. We have also emphasized that the lower energies of the SoLID experiment can be exploited to separate dimension- 6 from dimension-8 effects when combined with high invariantmass LHC data. To demonstrate this point we have presented example fits containing both dimension- 6 fourfermion operators and their dimension-eight extensions. Combined fits of LHC data and projected SoLID and P2 data break degeneracies between dimension-six and dimension-eight effects and tighten bounds on individual Wilson coefficients considerably.

\section{ACKNOWLEDGMENTS}

We thank K. Kumar and P. Souder for motivating this work and for helpful discussions. R. B. is supported by the DOE Contract No. DE-AC02-06CH11357. F. P. and D. W. are supported by the DOE Grants No. DE-FG0291ER40684 and No. DE-AC02-06CH11357. The U.S. Government retains for itself, and others acting on its behalf, a paid-up nonexclusive, irrevocable worldwide license in said article to reproduce, prepare derivative works, distribute copies to the public, and perform publicly and display publicly, by or on behalf of the Government. 
[1] W. Buchmuller and D. Wyler, Nucl. Phys. B268, 621 (1986).

[2] C. Arzt, M. B. Einhorn, and J. Wudka, Nucl. Phys. B433, 41 (1995).

[3] B. Grzadkowski, M. Iskrzynski, M. Misiak, and J. Rosiek, J. High Energy Phys. 10 (2010) 085.

[4] C. W. Murphy, J. High Energy Phys. 10 (2020) 174.

[5] H. L. Li, Z. Ren, J. Shu, M. L. Xiao, J. H. Yu, and Y. H. Zheng, arXiv:2005.00008 [Phys. Rev. D (to be published)].

[6] Z. Han and W. Skiba, Phys. Rev. D 71, 075009 (2005).

[7] C. Y. Chen, S. Dawson, and C. Zhang, Phys. Rev. D 89, 015016 (2014).

[8] J. Ellis, V. Sanz, and T. You, J. High Energy Phys. 07 (2014) 036.

[9] J. D. Wells and Z. Zhang, Phys. Rev. D 90, 033006 (2014).

[10] A. Falkowski and F. Riva, J. High Energy Phys. 02 (2015) 039.

[11] V. Cirigliano, W. Dekens, J. de Vries, and E. Mereghetti, Phys. Rev. D 94, 034031 (2016).

[12] J. de Blas, M. Ciuchini, E. Franco, S. Mishima, M. Pierini, L. Reina, and L. Silvestrini, J. High Energy Phys. 12 (2016) 135.

[13] C. Hartmann, W. Shepherd, and M. Trott, J. High Energy Phys. 03 (2017) 060.

[14] A. Falkowski, M. Gonzlez-Alonso, and K. Mimouni, J. High Energy Phys. 08 (2017) 123.

[15] A. Biekoetter, T. Corbett, and T. Plehn, SciPost Phys. 6, 064 (2019).

[16] C. Grojean, M. Montull, and M. Riembau, J. High Energy Phys. 03 (2019) 020.

[17] A. Pomarol and F. Riva, J. High Energy Phys. 01 (2014) 151.

[18] S. Di Vita, C. Grojean, G. Panico, M. Riembau, and T. Vantalon, J. High Energy Phys. 09 (2017) 069.

[19] E. da Silva Almeida, A. Alves, N. Rosa Agostinho, O. J. P. Éboli, and M. C. Gonzalez-Garcia, Phys. Rev. D 99, 033001 (2019).

[20] J. Ellis, C. W. Murphy, V. Sanz, and T. You, J. High Energy Phys. 06 (2018) 146.

[21] N. P. Hartland, F. Maltoni, E. R. Nocera, J. Rojo, E. Slade, E. Vryonidou, and C. Zhang, J. High Energy Phys. 04 (2019) 100.

[22] I. Brivio, S. Bruggisser, F. Maltoni, R. Moutafis, T. Plehn, E. Vryonidou, S. Westhoff, and C. Zhang, J. High Energy Phys. 02 (2020) 131.

[23] S. van Beek, E. R. Nocera, J. Rojo, and E. Slade, SciPost Phys. 7, 070 (2019).

[24] R. Aoude, T. Hurth, S. Renner, and W. Shepherd, J. High Energy Phys. 12 (2020) 113.

[25] J. Ellis, M. Madigan, K. Mimasu, V. Sanz, and T. You, J. High Energy Phys. 04 (2021) 279.

[26] R. Contino, A. Falkowski, F. Goertz, C. Grojean, and F. Riva, J. High Energy Phys. 07 (2016) 144.

[27] D. Liu, A. Pomarol, R. Rattazzi, and F. Riva, J. High Energy Phys. 11 (2016) 141.

[28] C. Degrande, J. High Energy Phys. 02 (2014) 101.
[29] C. Hays, A. Martin, V. Sanz, and J. Setford, J. High Energy Phys. 02 (2019) 123.

[30] B. Bellazzini and F. Riva, Phys. Rev. D 98, 095021 (2018).

[31] J. Ellis, S.-F. Ge, H.-J. He, and R.-Q. Xiao Rui, Sci. China Phys. Mech. Astron. 64, 221062 (2021).

[32] S. Alioli, R. Boughezal, E. Mereghetti, and F. Petriello, Phys. Lett. B 809, 135703 (2020).

[33] C. W. Murphy, J. High Energy Phys. 04 (2021) 101.

[34] C. Hays, A. Helset, A. Martin, and M. Trott, J. High Energy Phys. 11 (2020) 087.

[35] J. Ellis, H. J. He, and R. Q. Xiao, Sci. China Phys. Mech. Astron. 64, 221062 (2021).

[36] D. Wang et al. (PVDIS Collaboration), Nature (London) 506, 67 (2014).

[37] D. Wang, K. Pan, R. Subedi, Z. Ahmed, K. Allada, K. A. Aniol, D. S. Armstrong, J. Arrington, V. Bellini, R. Beminiwattha et al., Phys. Rev. C 91, 045506 (2015).

[38] D. Becker, R. Bucoveanu, C. Grzesik, K. Imai, R. Kempf, K. Imai, M. Molitor, A. Tyukin, M. Zimmermann, D. Armstrong et al., Eur. Phys. J. A 54, 208 (2018).

[39] S. Dawson, P. P. Giardino, and A. Ismail, Phys. Rev. D 99, 035044 (2019).

[40] S. Alte, M. König, and W. Shepherd, J. High Energy Phys. 07 (2019) 144.

[41] R. Boughezal, F. Petriello, and D. Wiegand, Phys. Rev. D 101, 116002 (2020).

[42] R. Boughezal, C. Y. Chen, F. Petriello, and D. Wiegand, Phys. Rev. D 103, 055015 (2021).

[43] P. A. Zyla et al. (Particle Data Group), Prog. Theor. Exp. Phys. 2020, 083 C01 (2020).

[44] A. Denner, Fortschr. Phys. 41, 307 (1993).

[45] The SOLID Collaboration, 'SoLID (Solenoidal Large Intensity Device) Updated Preliminary Conceptual Design Report, https://hallaweb.jlab.org/12GeV/SoLID/files/solidprecdr-Nov2019.pdf.

[46] Addendum for PAC35 Precision Measurement of Parityviolation in Deep Inelastic Scattering Over a Broad Kinematic Range (2009).

[47] T. Hobbs and W. Melnitchouk, Phys. Rev. D 77, 114023 (2008).

[48] S. Mantry, M. J. Ramsey-Musolf, and G. F. Sacco, Phys. Rev. C 82, 065205 (2010).

[49] M. R. Buckley and M. J. Ramsey-Musolf, Phys. Lett. B 712, 261 (2012).

[50] M. González-Alonso and M. J. Ramsey-Musolf, Phys. Rev. D 87, 055013 (2013).

[51] M. Aaboud et al. (ATLAS Collaboration), J. High Energy Phys. 10 (2017) 182.

[52] G. Aad et al. (ATLAS Collaboration), J. High Energy Phys. 08 (2016) 009.

[53] P. L. Anthony et al. (SLAC E158 Collaboration), Phys. Rev. Lett. 95, 081601 (2005).

[54] C. S. Wood, S. C. Bennett, D. Cho, B. P. Masterson, J. L. Roberts, C. E. Tanner, and C. E. Wieman, Science 275, 1759 (1997).

[55] D. Androic, D. S. Armstrong, A. Asaturyan, T. Averett, J. Balewski, K. Bartlett, J. Beaufait, R. S. Beminiwattha, 
J. Benesch and F. Benmokhtar et al., EPJ Web Conf. 137, 08005 (2017).

[56] E. E. Jenkins, A. V. Manohar, and M. Trott, J. High Energy Phys. 10 (2013) 087.

[57] E. E. Jenkins, A. V. Manohar, and M. Trott, J. High Energy Phys. 01 (2014) 035.
[58] R. Alonso, E. E. Jenkins, A. V. Manohar, and M. Trott, J. High Energy Phys. 04 (2014) 159.

[59] A. A. Akhundov, D. Y. Bardin, and T. Riemann, Nucl. Phys. B276, 1 (1986).

[60] W. Beenakker and W. Hollik, Z. Phys. C 40, 141 (1988). 